\title{
A review of tinnitus
}

Aaron A Esmaili, John Renton

\section{Background \\ Tinnitus is one of the most common otological symptoms. In recent times our understanding of tinnitus has significantly progressed. Tinnitus may be defined as conscious awareness of a sound in the absence of an external auditory stimulus. People with tinnitus almost always seek the attention of their general practitioner, who is best placed to assess, investigate and provide appropriate counselling.}

\section{Objective}

The aim of this article is to define and provide guidelines on causes, assessment, appropriate investigation and management of tinnitus.

\section{Discussion}

Tinnitus may be categorised as subjective, objective, primary or secondary. The assessment of tinnitus begins by determining which of these types with the patient presents with. All patients with tinnitus warrant formal audiometric assessment, and a proportion will warrant further imaging. Management requires treatment of reversible causes. Those with subjective, bothersome chronic tinnitus require tinnitus-specific therapies.
TINNITUS MAY BE DEFINED as conscious awareness of a sound in the absence of an external auditory stimulus. At least two-thirds of Australians have reported suffering from noticeable tinnitus at some point in their lives. ${ }^{1}$ Clinical care examples are given in Cases 1-3.

\section{Definitions}

Tinnitus may be categorised as subjective, objective, primary or secondary (Table 1). Tinnitus is further classified according to duration. One must also differentiate between bothersome tinnitus and nonbothersome tinnitus because this will influence how active the treating general practitioner (GP) needs to be about management.

\section{Causes}

Almost any insult to the auditory pathway can result in tinnitus. Several non-auditory conditions can also lead to tinnitus. Therefore, many organic states can lead to the experience of tinnitus. The most common and potentially reversible causes are outlined in Table 2.

\section{Assessment}

A directed history and examination is required when a patient presents with tinnitus.

\section{History}

- Timeline: When did the experience of tinnitus begin? Duration will determine whether tinnitus is in the acute or chronic state.

- Character: Obtain a description of the sound. Is it constant or pulsatile? Pulsatile tinnitus raises the possibility of a vascular lesion, such as a glomus tumour.

- Is the tinnitus unilateral or bilateral? Unilateral tinnitus raises the possibility of a focal lesion, such as a vestibular schwannoma.
- Are there any associated otological symptoms (eg otalgia, otorrhoea, vertigo)? Such symptoms raise the possibility of secondary tinnitus. Vertigo may be associated with inner ear disease (Table 2). ${ }^{2}$

- Is there any associated hearing loss? If so, is this sudden in onset? Sudden onset sensorineural hearing loss (SNHL) requires prompt management with steroids. ${ }^{3}$ Patients with sudden onset SNHL present with an acute subjective hearing loss in one or both ears. A tuning fork test will help differentiate conductive from sensorineural hearing loss. When there is sudden-onset SNHL, pure tone audiogram should be requested promptly, steroid therapy commenced, and the patient referred to an otolaryngologist urgently. Given unilateral tinnitus and hearing loss may raise the possibility of a focal lesion, magnetic resonance imaging (MRI) is often part of the work-up.

- Is there a history of noise exposure, whether occupational or social? This can lead to hearing loss and tinnitus. ${ }^{4}$

- Is the patient on any medication? Some medications (Table 2) can lead to hearing loss and tinnitus. ${ }^{5}$

- Is the tinnitus bothersome or nonbothersome? Some people have a very negative response to tinnitus, whereas others are not bothered by it.

- Associated anxiety or depression: It is important to identify those patients who are significantly distressed by tinnitus and have apparent symptoms of depression or anxiety. A link between tinnitus and comorbid psychological disorder has been demonstrated, ${ }^{2}$ and a high prevalence of anxiety and depression is seen in tinnitus sufferers.

\section{Examination}

Routine cranial nerve and otological examination is required. This is further guided by the history. A tuning fork assessment will provide a good indication 


\section{Table 1. Tinnitus definitions}

\begin{tabular}{ll}
\hline Type of tinnitus & Definition \\
\hline Subjective tinnitus & Only the affected individual can hear the sound \\
\hline Objective tinnitus & $\begin{array}{l}\text { The sound can also be heard by the examiner (eg crepitus of temporomandibular } \\
\text { joint, bruit of vascular malformation) }\end{array}$ \\
\hline Pulsatile tinnitus & $\begin{array}{l}\text { Tinnitus that is described as producing sound of regular pulsations. This may be } \\
\text { subjective or objective }\end{array}$ \\
\hline Primary tinnitus & $\begin{array}{l}\text { Tinnitus that is idiopathic and may or may not be associated with sensorineural } \\
\text { hearing loss (SNHL). The SNHL should be symmetrical }\end{array}$ \\
\hline Secondary tinnitus & \begin{tabular}{l} 
Tinnitus associated with a specific underlying cause (other than symmetrical SNHL) \\
\hline Acute or recent onset tinnitus
\end{tabular} \\
\hline Chronic tinnitus & Apparent for $<6$ months \\
\hline
\end{tabular}

of the degree and type of hearing loss. For this test, a $512 \mathrm{~Hz}$ tuning fork is typically used. The GP should first conduct a Weber test, whereby the tuning fork is placed in the middle of the patient's forehead. A normal test is demonstrated when the patient hears the sound equally in both ears. If the sound lateralises to a specific ear, the patient may have a conductive loss in that ear or a sensorineural loss in the contralateral ear. A Rinne test is then performed, whereby the tuning fork is placed first on the mastoid (bone conduction), then held vertically in front of the auricle (air conduction), and the patient is asked which is louder. A normal (positive Rinne) test is demonstrated when air conduction is louder than bone conduction. If the sound is louder with the tuning fork on the mastoid (bone conduction), this indicates a conductive hearing loss. Otoscopy helps to identify any abnormalities in the external and middle ear and help determine secondary causes of tinnitus. Finally, areas of the head and neck, including the carotid and periauricular regions, should be auscultated, especially if the patient reports pulsatile tinnitus. Tympanometers may be available in some general practice settings and, in this situation, obtaining a tympanogram may help assess middle ear status and eustachian tube function.

\section{Investigation}

\section{Audiology}

A formal audiology assessment is recommended for all patients presenting with tinnitus. This is because a large proportion of patients with tinnitus have some degree of hearing loss. ${ }^{6} \mathrm{~A}$ formal audiological assessment will provide a pure tone audiogram (PTA) and tympanogram. A PTA provides information about whether the patient has conductive hearing loss or SNHL. Conductive hearing loss almost always indicates a secondary cause for tinnitus. SNHL may indicate a primary or secondary cause. A tympanogram provides information about middle ear status and eustachian tube function.

\section{Imaging}

The American Academy of Otolaryngology - Head and Neck Surgery guideline ${ }^{2}$ suggest that imaging of the head and neck is indicated only if the patient has:

- unilateral tinnitus, which may suggest a focal lesion; therefore imaging is warranted

- pulsatile tinnitus, which may suggest a vascular anomaly; this is best investigated by computed tomography (CT) angiography in the general practice setting

- asymmetrical hearing loss, defined as $\geq 10 \mathrm{~dB}$ difference between the two sides in three or more frequencies; such patients will need an MRI of the cerebellopontine angle and internal acoustic meatus (MRI-CPA/IAM) to rule out a vestibular schwannoma

- focal neurological deficit

- otosclerosis, which often occurs in the third to fifth decade of life, and presents with a slowly progressive hearing loss and often with coexisting tinnitus - a tuning fork exam will suggest a conductive hearing loss, which can be confirmed by audiometry; in this situation, CT of the temporal bone is warranted.

CT of the temporal bone may also be indicated in the setting of trauma to the head and if cholesteatoma is suspected.

\section{Management}

Management of secondary tinnitus is centred on identification of the underlying cause and, where possible, reversal of the underlying condition. After thorough history-taking, clinical examination and appropriate investigation, the tinnitus may be categorised as primary. In a patient with primary tinnitus with or without SNHL, the GP needs to determine whether the tinnitus is bothersome and chronic. If it is not, then reassurance may be all that is needed. However, if it is bothersome, the following strategies may be considered. 


\section{Table 2. Common secondary causes of tinnitus}

External ear

- Cerumen impaction

- Otitis externa

Middle ear

- Otosclerosis

- Otitis media

- Cholesteatoma

Inner ear

- Vestibular schwannoma

- Meniere's disease

- Cochleitis/neuritis

- Ototoxic medications:

- antibiotics (eg aminoglycosides and vancomycin)

- cancer medications

- diuretics (eg frusemide)

- Aspirin or other salicylates

Non-auditory cause

- Vascular anomalies (present as pulsatile tinnitus)

- Myoclonus, such as stapedial myoclonus

- Nasopharyngeal carcinoma (can cause unilateral otitis media with effusion, leading to hearing loss and tinnitus)

\section{Hearing aids}

In the presence of SNHL that is bothersome, the GP should consider referring the patient for hearing aid assessment. Even in the absence of marked hearing loss, hearing aids can provide some relief. ${ }^{2}$ Hearing aids amplify peripheral and objective sounds and, treats the patient's tinnitus by making them less noticeable. ${ }^{7}$

\section{Sound therapy}

Sound therapy is normally prescribed by an audiologist, either alone or in combination with hearing aids, given some hearing aids have an inbuilt sound generator. Sound therapy alone can also be prescribed by the GP. The principle is that an external sound is provided to the auditory centre, the patient focuses on this sound and, therefore, has a reduced perception of tinnitus and a sense of relief. The patient can use their own music player or hearing aid, and there are also devices specifically marketed for patients with tinnitus. ${ }^{8}$

\section{Cognitive behavioural therapy}

It is widely recognised that tinnitus can cause emotional disturbance, which in turn stimulates the sympathetic nervous system and can increase the volume of tinnitus. ${ }^{2}$ Anxiety and depression can result. Cognitive behavioural therapy (CBT) was originally structured to treat anxiety and depression, and therefore can be effective in treating a patient's response to tinnitus. CBT encourages the patient to examine thought processes that may result in distress and negativity, and teaches skills to restructure these thoughts to bring about positivity. The GP, if appropriately trained, may provide such counselling or refer the patient to a psychologist with an interest in tinnitus.

A combination of treatment options may be necessary to allow the patient to habituate - in other words, to not be bothered by the noise they hear.

GPs should avoid prescribing antidepressants, anticonvulsants or anxiolytics for tinnitus, as there is no evidence for their effectiveness in treating tinnitus, and because of adverse effects. ${ }^{2,9}$ A variety of complementary medicines have been trialled in patients, but again there is no evidence for their use. ${ }^{2}$

\section{Conclusion}

Tinnitus is common and has many varied causes. After careful history-taking and examination, tinnitus can be classified as either primary or secondary. The goal for managing primary, bothersome tinnitus is to implement strategies to allow the patient to habituate. The aim for secondary tinnitus is to investigate and manage the underlying cause (Figure 1).

\section{CASE 1}

A man aged 79 years presents to you with bilateral tinnitus. The tinnitus has been apparent for five years, but has increased in volume over the past six months. The patient had worked near aircraft but is now retired. History and examination are otherwise unremarkable. A PTA reveals a symmetrical sloping hearing loss with dip at $4000 \mathrm{~Hz}$ (consistent with presbycusis, with associated noise-induced hearing loss). You refer the patient to an audiologist for hearing aids. He returns to inform you that not only is his hearing better, his tinnitus is less apparent when he uses his hearing aids.

\section{CASE 2}

A woman aged 44 years presents to you with bilateral tinnitus. You are aware that she has a history of anxiety and depression. History and examination are otherwise normal; therefore, you make the diagnosis of primary tinnitus. She informs you that the tinnitus is bothering her, affecting her sleep and marriage. A PTA reveals bilateral mild SNHL. This patient warrants multimodality treatment with hearing aids and counselling.

\section{CASE 3}

A woman aged 54 years presents to you with unilateral tinnitus on the right side. She also informs you that she feels her hearing has decreased on that side. Otoscopy is unremarkable. Tuning fork testing reveals Weber lateralising to the left and Rinne test positive bilateral (air conduction $>$ bone conduction), which is consistent with a right-sided SNHL. Pure tone audiometry reveals findings consistent with this. This patient warrants imaging. You organise an MRI-CPA/IAM, which reveals a right-sided vestibular schwannoma. The patient is referred to an ear, nose and throat surgeon for further management.

\section{Authors}

Aaron A Esmaili BPharm (Hon), MBBS (Hon), ENT Service Registrar, Department of Otolaryngology, Head and Neck Surgery, Sir Charles Gairdner Hospital, Nedlands, WA. aesmaili2@gmail.com John Renton MD, FACS, Neuro-otology Fellow, Sir Charles Gairdner Hospital, Nedlands, WA

Competing interests: None. 


\section{Tinnitus}

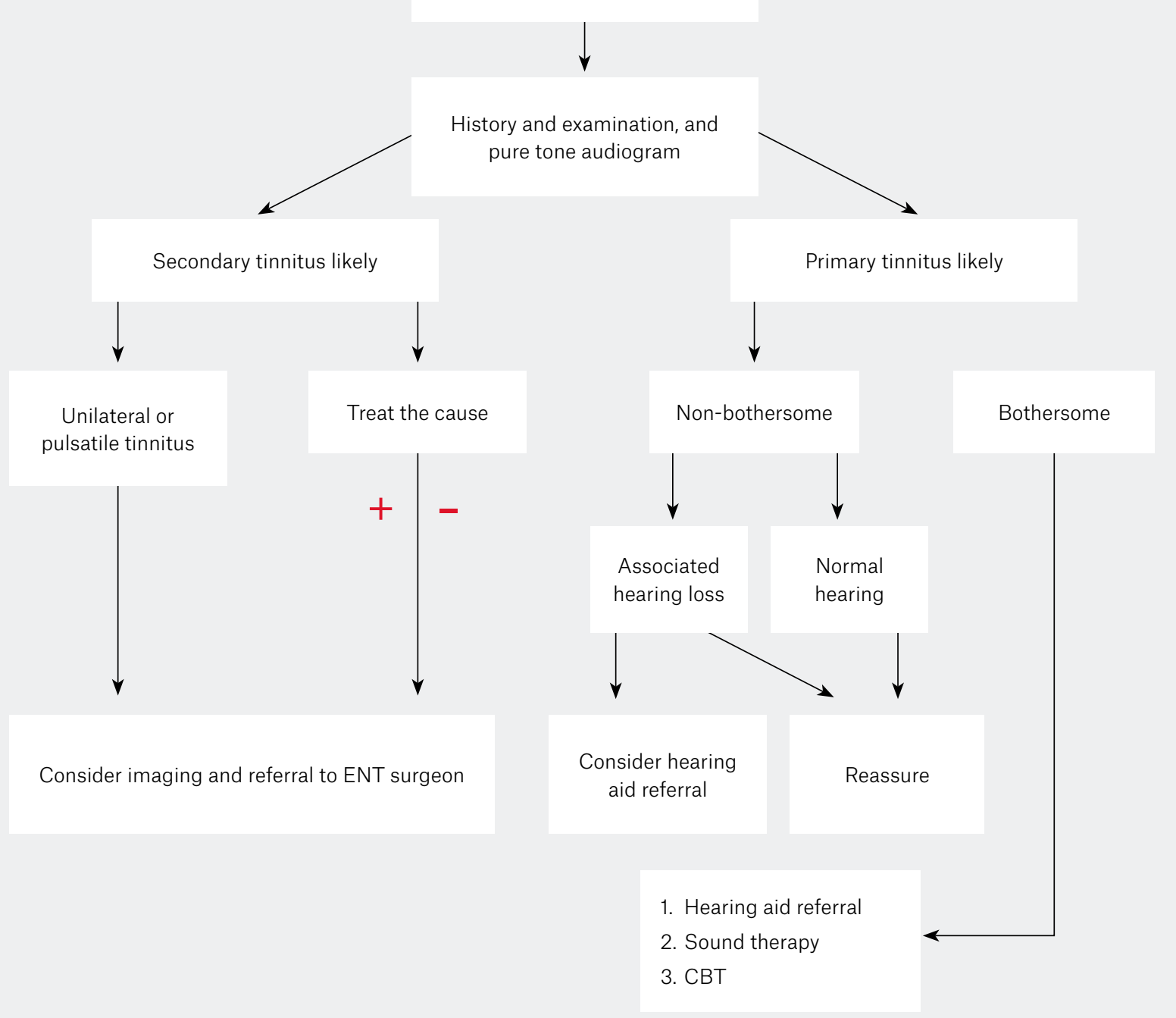

Figure 1. Suggested assessment and management pathway in patients with tinnitus CBT, cognitive behavioural therapy; ENT, ear, nose and throat

Provenance and peer review: Not commissioned, externally peer reviewed.

\section{References}

1. Sindhusake D, Mitchell P, Newall P, Golding M, Rochtchina E, Rubin G. Prevalence and characteristics of tinnitus in older adults: The Blue Mountains Hearing Study. Int J Audiol 2003; 42(5):289-94.

2. Tunkel DE, Bauer CA, Sun GH, et al. Clinical practice guideline: Tinnitus. Otolaryngol Head Neck Surg 2014;151(2 Suppl):S1-S40.

3. Lavigne P, Lavigne F, Saliba I. Intratympanic corticosteroids injections: A systematic review of literature. Eur Arch Otorhinolaryngol 2016;273(9):2271-78. doi: 10.1007/s00405-0153689-3.
4. Hickox AE, Liberman MC. Is noise-induced cochlear neuropathy key to the generation of hyperacusis or tinnitus? J Neurophysiology 2014; 111(3) 552-64.

5. Ganesan P, Schmeidge J, Manchaiah V, Swapna S, Dhandayutham S, Kothoandaraman PP. Ototoxicity: A challenge in diagnosis and treatment. J Audiol Oto. 2018; doi 10.7874/ jao.2017.00360 [epub ahead of print].6.

6. Hoare DJ, Edmondson-Jones M, Sereda M, Akeroyd MA, Hall D. Amplification with hearing aids for patients with tinnitus and co-existing hearing loss. Cochrane Database Syst Rev 2014;(1):CD010151. doi: 10.1002/14651858. CD010151.pub2.

7. Searchfield GD, Kaur M, Martin WH. Hearing aids as an adjunct to counseling: Tinnitus patients who choose amplification do better than those that don't. Int J Audiol 2010;49(8):574-79. doi: 10.3109/14992021003777267.

8. Davis PB, Paki B, Hanley PJ. Neuromonics Tinnitus Treatment: Third clinical trial. Ear Hear 2007;28(2):242-59.

9. Baldo P, Doree C, Molin P, McFerran D, Cecco S. Antidepressants for patients with tinnitus. Cochrane Database Syst Rev 2012;(9):CD003853. doi: 10.1002/14651858. CD003853.pub3. 